\title{
A Novel Numerical Method of Two-Dimensional Fredholm Integral Equations of the Second Kind
}

\author{
Yanying Ma, Jin Huang, and Hu Li \\ School of Mathematical Sciences, University of Electronic Science and Technology of China, Chengdu 611731, China \\ Correspondence should be addressed to Yanying Ma; ma_yan_ying@126.com
}

Received 23 April 2015; Accepted 12 July 2015

Academic Editor: Tadeusz Kaczorek

Copyright (C) 2015 Yanying Ma et al. This is an open access article distributed under the Creative Commons Attribution License, which permits unrestricted use, distribution, and reproduction in any medium, provided the original work is properly cited.

\begin{abstract}
A novel numerical method is developed for solving two-dimensional linear Fredholm integral equations of the second kind by integral mean value theorem. In the proposed algorithm, each element of the generated discrete matrix is not required to calculate integrals, and the approximate integral operator is convergent according to collectively compact theory. Convergence and error analyses of the approximate solution are provided. In addition, an algorithm is given. The reliability and efficiency of the proposed method will be illustrated by comparison with some numerical results.
\end{abstract}

\section{Introduction}

This paper proposes a novel numerical method based on integral mean value theorem (IMVT) for solving twodimensional linear Fredholm integral equations (FIE) of the second kind. The linear integral equation is considered as

$$
u-K u=f,
$$

where linear integral operator $K$ is defined as

$$
\begin{array}{r}
(K u)(x, y)=\int_{c}^{d} \int_{a}^{b} k(x, y, s, t) u(s, t) d s d t, \\
\quad(x, y) \in D,
\end{array}
$$

$f(x, y)$ and $k(x, y, s, t)$ are given continuous functions defined on $D=[a, b] \times[c, d]$ and $E=D \times D$, respectively, and $u(x, y)$ is an unknown function on $D$.

Equation (1) is a useful tool for a large amount of modeling problems which arise in computer graphics manipulations [1], aerodynamics, fracture mechanics, and electromagnetic scattering $[2,3]$. Their historical development is closely related to the solutions of boundary value problems. Generally, the boundary value problems of the differential equations are converted to integral equations [4, 5]. For example, Laplace equation of boundary conditions is reduced to FIE of the second kind by direct boundary element method [5].

There exist many methods dealing with one-dimensional integral equation $[1,2,4,6-8]$. However, high dimensional problem is still a challenge, a few numerical approaches dealing with high dimensional problems [9-22]. The generically significant numerical methods include collocation method [2, 9-15], Galerkin method [16, 19], and the wavelets method $[17,20]$. In $[9,10,13]$, several authors used orthogonal polynomials as the collocation method's basis functions, instead of polynomials, block-pulse functions (BFS) [2] and radial basis functions [11-14], and modification of hat functions (2D-MHFs) [15]. Furthermore, the authors compared the traditional collocation method by the orthogonal polynomials with radial basis functions (RBF) methods [14]. In [19], the authors proposed Richardson extrapolation of iterated discrete Galerkin method to achieve better accuracy. In [16], researchers presented some orthogonal polynomials as Galerkin method's basis functions to solve the linear FIE of the second kind. Both of the collocation method and Galerkin method belong to the projection method, and the key is to come up with good basis functions. 
Unlike the projection method, integral mean value method was proposed for one-dimensional integrals [7] and multiple integrals [18]. They transform integral equations to nonlinear systems of equations without any basis functions. Based on IMVT, they solved systems by iterative method. However, convergence analysis is not given in those papers. This paper introduces a new method by changing FIE to systems of linear algebraic equations through IMVT. Each element of generated discrete matrix does not need calculation of integrals, which has an advantage in decreasing the computation, and approximation integral operator is convergent under collectively compact theory. Finally, an algorithm is implemented.

This paper is organized into four sections. In Section 2, a numerical method based on the idea of IMVT is given. In Section 3, the convergence and error analyses of the numerical solution are provided. In Section 4, numerical examples are carried out.

\section{A Numerical Method}

We review the two-dimensional IMVT as follows.

Lemma 1 (IMVT for double integrals [18]). If $g(x, y)$ is continuous on $[a, b] \times[c, d]$, then there exists a point $(\alpha, \beta) \in$ $[a, b] \times[c, d]$, such that

$$
Q(g)=\iint_{D} g(x, y) d x d y=(b-a)(d-c) g(\alpha, \beta) .
$$

Let $h_{1}=(b-a) / m$ and $h_{2}=(d-c) / n, m, n \in N$, and $x_{i}=a+i h_{1}(i=0, \ldots, m-1)$ and $y_{j}=c+j h_{2}(j=0, \ldots, n-1)$. By (3), a sequence of quadrature formula can be constructed as follows:

$$
\begin{aligned}
Q\left(g, \alpha_{i}, \beta_{j}\right) & =\sum_{i=0}^{m-1} \sum_{j=0}^{n-1} \int_{x_{i}}^{x_{i+1}} \int_{y_{j}}^{y_{j+1}} g(x, y) d y d x \\
& =h_{1} h_{2} \sum_{i=0}^{m-1} \sum_{j=0}^{n-1} g\left(x_{i}+h_{1} \alpha_{i}, y_{j}+h_{2} \beta_{j}\right),
\end{aligned}
$$

where $\alpha_{i}$ and $\beta_{j}$ are constants with $0 \leq \alpha_{i} \leq 1$ and $0 \leq \beta_{j} \leq$ 1. Once $\alpha_{i}$ and $\beta_{j}(i=0, \ldots, m-1, j=0, \ldots, n-1)$ are determined, (4) could be accurately calculated. According to (4), we have

$$
\begin{aligned}
& (K u)(x, y) \\
& =\sum_{i=0}^{m-1} \sum_{j=0}^{n-1} \int_{x_{i}}^{x_{i}+h_{1}} \int_{y_{j}}^{y_{j}+h_{2}} k(x, y, s, t) u(s, t) d s d t \\
& =h_{1} h_{2} \sum_{i=0}^{m-1} \sum_{j=0}^{n-1} k\left(x, y, x_{i}+h_{1} \alpha_{i}(x), y_{j}+h_{2} \beta_{j}(y)\right) \\
& \cdot u\left(x_{i}+h_{1} \alpha_{i}(x), y_{j}+h_{2} \beta_{j}(y)\right), \quad(x, y) \in D,
\end{aligned}
$$

where $\alpha_{i}(x)$ and $\beta_{j}(y)$ are functions that depend on $x$ and $y$ separately with $0 \leq \alpha_{i}(x) \leq 1$ and $0 \leq \beta_{j}(y) \leq 1(i=$ $0, \ldots, m-1$ and $j=0, \ldots, n-1)$. To simplify, $\alpha_{i}(x)$ and $\beta_{j}(y)$ are assumed to be constants, namely, $\alpha_{i}(x)=\alpha_{i}$ and $\beta_{j}(y)=$ $\beta_{j}$. Then an approximate integral equation can be formulated as follows:

$$
\begin{aligned}
& \left(K_{m n} u\right)(x, y) \\
& =h_{1} h_{2} \sum_{i=0}^{m-1} \sum_{j=0}^{n-1} k\left(x, y, x_{i}+h_{1} \alpha_{i}, y_{j}+h_{2} \beta_{j}\right) \\
& \cdot u\left(x_{i}+h_{1} \alpha_{i}, y_{j}+h_{2} \beta_{j}\right) .
\end{aligned}
$$

Thus, (1) is rewritten as

$$
\begin{gathered}
u_{m n}(x, y)-h_{1} h_{2} \sum_{i=0}^{m-1} \sum_{j=0}^{n-1} k\left(x, y, x_{i}+h_{1} \alpha_{i}, y_{j}+h_{2} \beta_{j}\right) \\
\cdot u_{m n}\left(x_{i}+h_{1} \alpha_{i}, y_{j}+h_{2} \beta_{j}\right)=f(x, y) .
\end{gathered}
$$

Let $x=x_{k}+h_{1} \alpha_{k}$ and $y=y_{l}+h_{2} \beta_{l}$ in (7); one can get the following linear system:

$$
\begin{aligned}
& u_{m n}\left(x_{k}+h_{1} \alpha_{k}, y_{l}+h_{2} \beta_{l}\right)=h_{1} h_{2} \sum_{i=0}^{m-1} \sum_{j=0}^{n-1} k\left(x_{k}\right. \\
& \left.+h_{1} \alpha_{k}, y_{l}+h_{2} \beta_{l}, x_{i}+h_{1} \alpha_{i}, y_{j}+h_{2} \beta_{j}\right) u_{m n}\left(x_{i}\right. \\
& \left.+h_{1} \alpha_{i}, y_{j}+h_{2} \beta_{j}\right)+f\left(x_{k}+h_{1} \alpha_{k}, y_{l}+h_{2} \beta_{l}\right)
\end{aligned}
$$

with $k=0, \ldots, m-1$ and $l=0, \ldots, n-1$. Hence, the approximate operator of (1) is

$$
u_{m n}-K_{m n} u_{m n}=f .
$$

Once we find the value of $u_{m n}\left(x_{k}+h_{1} \alpha_{k}, y_{l}+h_{2} \beta_{l}\right)$ in (8), we can get approximate solution of (1). In a word, we can obtain value of $u(x, y)$ at any point of the region $D$ by $u(x, y)=K u_{m n}+f(x, y)$.

When $\alpha_{i}=\beta_{j}=1 / 2$ in (8), the formula is considered as the midpoint rule for solving two-dimensional FIE.

\section{Convergence and Error Analysis}

In this section, we give the convergence analysis of $K_{m n}$. Furthermore, we prove the existence and uniqueness of solution in (9) and give the error analysis of the approximate solution.

Lemma 2. If $k(x, y, s, t)$ is continuous function on $E=D \times D$, then the norm of $K_{m n}$ is

$$
\begin{aligned}
& \left\|K_{m n}\right\|_{\infty} \\
& \quad=h_{1} h_{2} \max _{(x, y) \in D} \sum_{i=0}^{m-1} \sum_{j=0}^{n-1}\left|k\left(x, y, x_{i}+h_{1} \alpha_{i}, y_{j}+h_{2} \beta_{j}\right)\right| .
\end{aligned}
$$

Proof. For $\forall u(x, y) \in C([a, b] \times[c, d])$ and $\|u\|_{\infty} \leq 1$, one has 


$$
\begin{aligned}
\left\|K_{m n} u\right\|_{\infty} & =h_{1} h_{2} \max _{(x, y) \in D}\left|\sum_{i=0}^{m-1} \sum_{j=0}^{n-1} k\left(x, y, x_{i}+h_{1} \alpha_{i}, y_{j}+h_{2} \beta_{j}\right) u\left(x_{i}+h_{1} \alpha_{i}, y_{j}+h_{2} \beta_{j}\right)\right| \\
& \leq h_{1} h_{2} \max _{(x, y) \in D} \sum_{i=0}^{m-1} \sum_{j=0}^{n-1}\left|k\left(x, y, x_{i}+h_{1} \alpha_{i}, y_{j}+h_{2} \beta_{j}\right) u\left(x_{i}+h_{1} \alpha_{i}, y_{j}+h_{2} \beta_{j}\right)\right| \\
& \leq h_{1} h_{2} \max _{(x, y) \in D} \sum_{i=0}^{m-1} \sum_{j=0}^{n-1}\left|k\left(x, y, x_{i}+h_{1} \alpha_{i}, y_{j}+h_{2} \beta_{j}\right)\right| .
\end{aligned}
$$

Since kernel function $k(x, y, s, t)$ is continuous on $D \times D$, there exists $\left(x_{0}, y_{0}\right) \in D$ such that

$$
\begin{aligned}
& \sum_{i=0}^{m-1} \sum_{j=0}^{n-1}\left|k\left(x_{0}, y_{0}, x_{i}+h_{1} \alpha_{i}, y_{j}+h_{2} \beta_{j}\right)\right| \\
& \quad=h_{1} h_{2} \max _{(x, y) \in D} \sum_{i=0}^{m-1} \sum_{j=0}^{n-1}\left|k\left(x, y, x_{i}+h_{1} \alpha_{i}, y_{j}+h_{2} \beta_{j}\right)\right| .
\end{aligned}
$$

We choose $u_{0} \in C([a, b] \times[c, d])$ with $\left\|u_{0}\right\|_{\infty}=1$ and give

$$
\begin{aligned}
k & \left(x_{0}, y_{0}, x_{i}+h_{1} \alpha_{i}, y_{j}+h_{2} \beta_{j}\right) \\
& \cdot u_{0}\left(x_{i}+h_{1} \alpha_{i}, y_{j}+h_{2} \beta_{j}\right) \\
& =k\left(x_{0}, y_{0}, x_{i}+h_{1} \alpha_{i}, y_{j}+h_{2} \beta_{j}\right),
\end{aligned}
$$

with $k=0, \ldots, m-1$ and $l=0, \ldots, n-1$.
On the other hand, we have

$$
\begin{aligned}
& \left\|K_{m n}\right\|_{\infty} \geq\left\|K_{m n} u_{0}\right\|_{\infty} \geq\left|\left(K_{m n} u_{0}\right)\left(x_{0}, y_{0}\right)\right| \\
& =h_{1} h_{2} \max _{(x, y) \in D} \sum_{i=0}^{m-1} \sum_{j=0}^{n-1}\left|k\left(x, y, x_{i}+h_{1} \alpha_{i}, y_{j}+h_{2} \beta_{j}\right)\right|,
\end{aligned}
$$

and the proof of Lemma 2 is completed.

Let $\alpha_{i}=\alpha(i=0, \ldots, m-1)$ and $\beta_{j}=\beta(j=0, \ldots, n-1)$ in (4), where $\alpha$ and $\beta$ are constants, such that

$$
Q_{m n}(g, \alpha, \beta)=h_{1} h_{2} \sum_{i=0}^{m-1} \sum_{j=0}^{n-1} g\left(x_{i}+h_{1} \alpha, y_{j}+h_{2} \beta\right),
$$

$$
0 \leq \alpha, \beta \leq 1
$$

The following theorem gives the convergence analysis of (15).

Theorem 3. Let $g(x, y) \in C([a, b] \times[c, d])$ and $g(x, y)$ satisfy Lipschitz condition; that is, $\exists L_{1}>0$ and $L_{2}>0$ :

$$
\begin{gathered}
\left\|g\left(x_{1}, y\right)-g\left(x_{2}, y\right)\right\|_{\infty} \leq L_{1}\left\|x_{1}-x_{2}\right\|_{\infty}, \\
\left\|g\left(x, y_{1}\right)-g\left(x, y_{2}\right)\right\|_{\infty} \leq L_{2}\left\|y_{1}-y_{2}\right\|_{\infty} .
\end{gathered}
$$

The quadrature operator $Q_{m n}(g, \alpha, \beta)$ is a uniform convergence sequence: namely, $\left\|Q_{m n}(g, \alpha, \beta)-Q(g)\right\|_{\infty} \rightarrow 0$, as $m, n \rightarrow$ $\infty$.

Proof. From (4) and (15), one can find

$$
\begin{aligned}
& \left\|Q_{m n}(g, \alpha, \beta)-Q(g)\right\|_{\infty}=\left\|Q_{m n}(g, \alpha, \beta)-Q\left(g, \alpha_{i}, \beta_{j}\right)\right\|_{\infty} \\
& \leq h_{1} h_{2} \sum_{i=0}^{m-1} \sum_{j=0}^{n-1}\left\|\left(g\left(x_{i}+h_{1} \alpha, y_{j}+h_{2} \beta\right)-g\left(x_{i}+h_{1} \alpha_{i}, y_{j}+h_{2} \beta_{j}\right)\right)\right\|_{\infty} \\
& \quad=h_{1} h_{2} \sum_{i=0}^{m-1} \sum_{j=0}^{n-1}\left\|g\left(x_{i}+h_{1} \alpha, y_{j}+h_{2} \beta\right)-g\left(x_{i}+h_{1} \alpha_{i}, y_{j}+h_{2} \beta\right)+g\left(x_{i}+h_{1} \alpha_{i}, y_{j}+h_{2} \beta\right)-g\left(x_{i}+h_{1} \alpha_{i}, y_{j}+h_{2} \beta_{j}\right)\right\|_{\infty} \\
& \quad \leq h_{1} h_{2} \sum_{i=0}^{m-1} \sum_{j=0}^{n-1}\left(L_{1} h_{1}\left\|\alpha-\alpha_{i}\right\|_{\infty}+L_{2} h_{2}\left\|\beta-\beta_{j}\right\|_{\infty}\right) \leq \frac{L_{1}(b-a)^{2}(d-c)}{m}+\frac{L_{2}(b-a)(d-c)^{2}}{n},
\end{aligned}
$$


where $0 \leq \alpha, \beta \leq 1$ and $0 \leq \alpha_{i}, \beta_{j} \leq 1$. When $m, n \rightarrow$ $\infty,\left\|Q_{m n}(g, \alpha, \beta)-Q(g)\right\|_{\infty} \rightarrow 0$. The proof of Theorem 3 is achieved.

Next we give the convergence analysis of (6).

Theorem 4. Let $k(x, y, s, t) \in C(D \times D), u(x, y) \in C(D)$ and $\exists L_{1}>0, L_{2}>0$ satisfy the following conditions such that

$$
\begin{aligned}
& \left\|k\left(x, y, s_{1}, t_{1}\right)-k\left(x, y, s_{2}, t_{2}\right)\right\|_{\infty} \\
& \quad \leq L_{1}\left(\left\|s_{1}-s_{2}\right\|_{\infty}+\left\|t_{1}-t_{2}\right\|_{\infty}\right), \\
& \left\|u\left(s_{1}, t_{1}\right)-u\left(s_{2}, t_{2}\right)\right\|_{\infty} \\
& \quad \leq L_{2}\left(\left\|s_{1}-s_{2}\right\|_{\infty}+\left\|t_{1}-t_{2}\right\|_{\infty}\right) .
\end{aligned}
$$

Then quadrature operator $\left(K_{m n} u\right)(x, y)$ is a uniform convergence sequence: namely, $\left\|\left(K_{m n} u\right)(x, y)-(K u)(x, y)\right\|_{\infty} \rightarrow 0$, as $m, n \rightarrow \infty$.

Proof. From (5) and (6), we have

$$
\begin{aligned}
& \left\|\left(K_{m n} u\right)(x, y)-(K u)(x, y)\right\|_{\infty} \\
& \leq h_{1} h_{2} \sum_{i=0}^{m-1} \sum_{j=0}^{n-1} \| k\left(x, y, x_{i}+h_{1} \alpha_{i}, y_{j}+h_{2} \beta_{j}\right) \\
& \cdot u\left(x_{i}+h_{1} \alpha_{i}, y_{j}+h_{2} \beta_{j}\right) \\
& -k\left(x, y, x_{i}+h_{1} \alpha_{i}(x), y_{j}+h_{2} \beta_{j}(y)\right) \\
& \cdot u\left(x_{i}+h_{1} \alpha_{i}(x), y_{j}+h_{2} \beta_{j}(y)\right) \|_{\infty} \\
& =h_{1} h_{2} \sum_{i=0}^{m-1} \sum_{j=0}^{n-1} \| k\left(x, y, x_{i}+h_{1} \alpha_{i}, y_{j}+h_{2} \beta_{j}\right) \\
& \cdot u\left(x_{i}+h_{1} \alpha_{i}, y_{j}+h_{2} \beta_{j}\right) \\
& -k\left(x, y, x_{i}+h_{1} \alpha_{i}(x), y_{j}+h_{2} \beta_{j}(y)\right) \\
& \cdot u\left(x_{i}+h_{1} \alpha_{i}, y_{j}+h_{2} \beta_{j}\right) \\
& +k\left(x, y, x_{i}+h_{1} \alpha_{i}(x), y_{j}+h_{2} \beta_{j}(y)\right) \\
& \cdot u\left(x_{i}+h_{1} \alpha_{i}, y_{j}+h_{2} \beta_{j}\right) \\
& -k\left(x, y, x_{i}+h_{1} \alpha_{i}(x), y_{j}+h_{2} \beta_{j}(y)\right) \\
& \cdot u\left(x_{i}+h_{1} \alpha_{i}(x), y_{j}+h_{2} \beta_{j}(y)\right) \|_{\infty} \\
& \leq h_{1} h_{2} \sum_{i=0}^{m-1} \sum_{j=0}^{n-1} \| k\left(x, y, x_{i} \alpha_{i}, y_{j}+h_{2} \alpha_{j}, y_{j}+h_{2} \beta_{j}\right) \\
& \cdot k
\end{aligned}
$$

$$
\begin{aligned}
& -k\left(x, y, x_{i}+h_{1} \alpha_{i}(x), y_{j}+h_{2} \beta_{j}(y)\right) \\
& \cdot u\left(x_{i}+h_{1} \alpha_{i}, y_{j}+h_{2} \beta_{j}\right) \|_{\infty} \\
& +h_{1} h_{2} \sum_{i=0}^{m-1} \sum_{j=0}^{n-1} \| k\left(x, y, x_{i}+h_{1} \alpha_{i}(x), y_{j}+h_{2} \beta_{j}(y)\right) \\
& \cdot u\left(x_{i}+h_{1} \alpha_{i}, y_{j}+h_{2} \beta_{j}\right) \\
& -k\left(x, y, x_{i}+h_{1} \alpha_{i}(x), y_{j}+h_{2} \beta_{j}(y)\right) \\
& \cdot u\left(x_{i}+h_{1} \alpha_{i}(x), y_{j}+h_{2} \beta_{j}(y)\right) \|_{\infty} \leq h_{1} h_{2}\left(L_{1}\right. \\
& \left.\cdot \max _{(x, y) \in D}|u(x, y)|+L_{2} \max _{(x, y, s, t) \in D \times D}|k(x, y, s, t)|\right) \\
& \cdot \sum_{i=0}^{m-1} \sum_{j=0}^{n-1}\left(\left\|h_{1}\left(\alpha_{i}-\alpha_{i}(x)\right)\right\|_{\infty}\right. \\
& \left.+\left\|h_{2}\left(\beta_{j}-\beta_{j}(y)\right)\right\|_{\infty}\right) \leq\left(\frac{(b-a)^{2}(d-c)}{m}\right. \\
& \left.+\frac{(d-c)^{2}(b-a)}{n}\right)\left(L_{1} \max _{(x, y) \in D}|u(x, y)|+L_{2}\right. \\
& \left.\max _{(x, y, s, t) \in D \times D}|k(x, y, s, t)|\right),
\end{aligned}
$$

where $0 \leq \alpha_{i}, \beta_{j} \leq 1$ and $0 \leq \alpha_{i}(x), \beta_{j}(y) \leq 1$.

Thus, $\left\|\left(K_{m n} u\right)(x, y)-(K u)(x, y)\right\|_{\infty} \rightarrow 0$ as $m, n \rightarrow \infty$. The proof of Theorem 4 is finished.

Theorem 5. Based on the conditions of Theorem 4, quadrature operator $K_{m n}$ is collectivity compact convergence to K: namely, $K_{m n} \stackrel{c c}{\longrightarrow} K$, as $m, n \rightarrow \infty$.

Proof. Based on Theorem 4 , we can easily get $K_{m n} \stackrel{\text { P }}{\rightarrow} K$, as $m, n \rightarrow \infty$. And from [23], we only need to prove that $S=$ $\bigcup_{m, n=1}^{\infty}\left(K-K_{m n}\right) B$ (B is unit sphere) is a relatively compact set. We need to show that function in $S$ is uniformly bounded and equicontinuous by Ascoli-Arzela theorem. So $\forall u \in B$,

$$
\begin{aligned}
& \left|K_{m n} u\right| \leq \sum_{i=0}^{m-1} \sum_{j=0}^{n-1} h_{1} h_{2} \mid k\left(x, y, x_{i}+h_{1} \alpha_{i}, y_{j}+h_{2} \beta_{j}\right) \\
& \cdot u\left(x_{i}+h_{1} \alpha_{i}, y_{j}+h_{2} \beta_{j}\right) \mid \\
& \leq \max _{(x, y, s, t) \in D \times D}|k(x, y, s, t)| \cdot\|u\|_{\infty} \sum_{i=0}^{m-1} \sum_{j=0}^{n-1} h_{1} h_{2} \\
& \leq C(b-a)(d-c) \max _{(x, y, s, t) \in D \times D}|k(x, y, s, t)| .
\end{aligned}
$$


Hence, $K_{m n} u$ is uniformly bounded. Then $\forall\left(x_{1}, y_{1}\right)$, $\left(x_{2}, y_{2}\right) \in D$ :

$$
\begin{aligned}
& \left|K_{m n} u\left(x_{1}, y_{1}\right)-K_{m n} u\left(x_{2}, y_{2}\right)\right| \\
& \quad \leq C\|u\|_{\infty} \max _{(x, y) \in D}\left|k\left(x, y, x_{1}, y_{1}\right)-k\left(x, y, x_{2}, y_{2}\right)\right| .
\end{aligned}
$$

When $\sqrt{\left(x_{1}-x_{2}\right)^{2}+\left(y_{1}-y_{2}\right)^{2}} \rightarrow 0$, the right side of the above formula converges to zero independent of $u(x)$ and $n$. So $K_{m n} u$ is equicontinuous. We can obtain that $S=$ $\bigcup_{m, n=1}^{\infty}\left(K_{m n}\right) B$ is a relatively compact set. Furthermore, $S=$ $\bigcup_{m, n=1}^{\infty}\left(K-K_{m n}\right) B$ is a relatively compact set because $K$ is compact operator. We can obtain the desired result.

Theorem 6. If integral operator $K$ satisfies the conditions of Theorem 4 and 1 is not eigenvalue of (1), the solution of approximate equation (17) is convergent to the solution of (1).

Proof. First, we prove the existence and uniqueness of the solution in (9). From Theorem 5 and the properties of collectively compact operator convergence [23], we know $\left\|K^{2}-K_{m n} K\right\| \rightarrow 0$ and $\left\|K^{2}-K K_{m n}\right\| \rightarrow 0$, as $m, n \rightarrow \infty$. Then we use the following identity:

$$
\begin{aligned}
(I & \left.-K+K_{m n}\right)\left(I-K_{m n}\right)=I-K+\left(K K_{m n}-K_{m n}^{2}\right) \\
& =(I-K)\left[I+(I-K)^{-1}\left(K K_{m n}-K_{m n}^{2}\right)\right] \\
& =(I-K)\left[I-(I-K)^{-1}\left(K_{m n}^{2}-K K_{m n}\right)\right] .
\end{aligned}
$$

Therefore, there exist sufficiently large $m, n$ such that

$$
\left\|I-(I-K)^{-1}\left(K_{m n}^{2}-K K_{m n}\right)\right\|_{\infty}<1 .
$$

Thus, the right side of (22) is inverse, and the left side of (22) is also inverse. Furthermore,

$$
\begin{aligned}
\left(I-K_{m n}\right)^{-1}= & {\left[I-(I-K)^{-1}\left(K_{m n}^{2}-K K_{m n}\right)\right]^{-1} } \\
& \cdot(I-K)^{-1}\left(I-K+K_{m n}\right) .
\end{aligned}
$$

That is, there exists a unique solution of (9) and $\left(I-K_{m n}\right)^{-1}$ is uniformly bounded. From (1) and (9), we have

$$
\begin{aligned}
\left(I-K_{m n}\right)\left(u-u_{m n}\right) & =\left(I-K_{m n}\right) u-f \\
& =\left(I-K_{m n}\right) u-(I-K) u \\
& =\left(K-K_{m n}\right) .
\end{aligned}
$$

According to Theorem 4 and the fact that $\left(I-K_{m n}\right)^{-1}$ is uniformly bounded, the following inequality holds:

$$
\begin{aligned}
\left\|u-u_{m n}\right\|_{\infty} & \leq\left\|\left(I-K_{m n}\right)^{-1}\right\|_{\infty}\left\|\left(K-K_{m n}\right) u\right\|_{\infty} \\
& \longrightarrow 0, \quad \text { as } m, n \longrightarrow \infty .
\end{aligned}
$$

Thus, we obtain the desired result of Theorem 6 .
From Theorem 4, the error of the approximate solution in (9) can be estimated. So we can get a corollary as follows.

Corollary 7. Under the assumption of Theorem 4, the error of the approximate solution can be estimated:

$$
\begin{aligned}
& \left\|u_{m n}(x, y)-u(x, y)\right\|_{\infty} \leq\left(\frac{(b-a)^{2}(d-c)}{m}\right. \\
& \left.+\frac{(d-c)^{2}(b-a)}{n}\right)\left(L_{1} \max _{(x, y) \in D}|u(x, y)|\right. \\
& \left.+L_{2} \max _{(x, y, s, t) \in D \times D}|k(x, y, s, t)|\right) .
\end{aligned}
$$

\section{Numerical Examples}

Below we provide an improvement algorithm.

Step 1. Select $m, n, \alpha_{i}=\beta_{j}=\theta$, where $\theta$ is constant in (7). And choose a series of arbitrary $\theta_{p}$ such that $0 \leq \alpha=$ $\beta=\theta_{p} \leq 1,(p=0, \ldots, q)$.

Step 2. Solve linear system (8).

Step 3. Get an approximation $u_{m n}\left(x, y, \theta_{p}\right)$ from (7).

Step 4. Calculate the average value of $u_{m n}\left(x, y, \theta_{p}\right)$ as the final approximate solution:

$$
u_{m n}(x, y)=\sum_{p=0}^{q} \frac{u_{m n}\left(x, y, \theta_{p}\right)}{q+1} .
$$

To verify the algorithm developed above, three examples are studied. The error used is defined by

$$
\text { Absolute error }=\left|u_{m n}(x, y)-u(x, y)\right|,
$$

where $u_{m n}(x, y)$ and $u(x, y)$ denote the numerical and exact solution at the point $(x, y)$, respectively.

Example 1. Consider the following equation:

$$
u(x, y)=f(x, y)-\iint_{0}^{1} x y \exp (s+t) u(s, t) d s d t
$$

where $f(x, y)=\exp (-x-y)$ and $u(x, y)=\exp (-x-y)-$ $(1 / 2) x y,(x, y) \in([0,1) \times[0,1))$.

For the sake of simplicity, $\theta_{p}$ is given as $\theta_{p}=p / 10(p=$ $0,1, \ldots, 10)$ in Tables 1 and 2 . The first three columns of Table 1 display absolute error for $m=n=4,8,16$, respectively, and the approximate solution more accurate than the last columns of midpoint rule. In Table 2, the absolute error of present method compares with 2D-TFs method [10]. It can be concluded that the proposed method is more accurate, rapidly convergent than method [10]. And Figure 1 shows the surfaces of the absolute error in a square on three different situations. When $m<n$ with $\theta_{p}=p / 10(p=0,1, \ldots, 10)$, the absolute error surfaces have been obtained for $u_{m n}$, as depicted in Figure 1(a). Similarly Figures 1(b) and 1(c) 
TABLE 1: Absolute errors of Example 1, $q=10$.

\begin{tabular}{lcccc}
\hline$(x, y)=\left(1 / 2^{l}, 1 / 2^{l}\right)$ & $m=n=4$ & $m=n=8$ & $m=n=16$ & Midpoint rule \\
\hline$l=1$ & $2.6643 e-03$ & $6.8809 e-04$ & $1.7377 e-04$ & $2.3848 e-02$ \\
$l=2$ & $6.6084 e-04$ & $1.7702 e-04$ & $4.3443 e-05$ & $5.9619 e-03$ \\
$l=3$ & $1.6521 e-04$ & $4.3006 e-05$ & $1.0861 e-05$ & $1.4905 e-03$ \\
$l=4$ & $4.1303 e-05$ & $1.0751 e-05$ & $2.7152 e-06$ & $3.7262 e-04$ \\
$l=5$ & $1.0326 e-05$ & $2.6879 e-06$ & $6.7879 e-07$ & $9.3155 e-05$ \\
$l=6$ & $2.5814 e-06$ & $6.7196 e-07$ & $1.6970 e-07$ & $2.3289 e-05$ \\
\hline
\end{tabular}

TABLE 2: Errors result of Example 1, $q=10$.

\begin{tabular}{lcccc}
\hline$(x, y)$ & $m=n=16$ & $m=n=32$ & {$[10] m=n=16$} & {$[10] m=n=32$} \\
\hline$(0.2,0.2)$ & $2.5901 e-05$ & $6.4892 e-06$ & $9.0644 e-03$ & $9.3553 e-03$ \\
$(0.4,0.4)$ & $1.0362 e-04$ & $2.5957 e-05$ & $1.3283 e-02$ & $1.4788 e-02$ \\
$(0.6,0.6)$ & $2.3311 e-04$ & $5.8402 e-05$ & $1.5467 e-02$ & $6.6765 e-05$ \\
$(0.8,0.8)$ & $4.1442 e-04$ & $1.0383 e-05$ & $1.7192 e-02$ & $4.5846 e-03$ \\
\hline
\end{tabular}

TABLE 3: Absolute errors of Example 2, $q=10$.

\begin{tabular}{lcccc}
\hline$(x, y)=\left(1 / 2^{l}, 1 / 2^{l}\right)$ & $m=n=8$ & $m=n=16$ & Midpoint rule \\
\hline$l=1$ & $3.9568 e-04$ & $9.9521 e-05$ & $2.4919 e-05$ & $2.5252 e-03$ \\
$l=2$ & $2.0383 e-04$ & $5.1268 e-05$ & $1.2837 e-05$ & $1.3009 e-03$ \\
$l=3$ & $1.0249 e-04$ & $2.6029 e-05$ & $6.5174 e-06$ & $6.6045 e-04$ \\
$l=4$ & $5.2144 e-05$ & $1.3115 e-05$ & $3.2839 e-06$ & $3.3279 e-04$ \\
$l=5$ & $2.6173 e-05$ & $6.5831 e-06$ & $1.6484 e-06$ & $1.6704 e-04$ \\
$l=6$ & $1.3112 e-05$ & $3.2980 e-06$ & $8.2579 e-07$ & $8.3683 e-05$ \\
\hline
\end{tabular}

TABLE 4: Absolute errors of Example 2, $q=10$.

\begin{tabular}{lcccc}
\hline$(x, y)$ & $m=n=16$ & $m=n=32$ & {$[10] m=n=16$} & {$[10] m=n=32$} \\
\hline$(0.2,0.2)$ & $8.8102 e-06$ & $2.5841 e-06$ & $9.9576 e-03$ & $9.5377 e-03$ \\
$(0.4,0.4)$ & $1.7201 e-05$ & $5.0452 e-06$ & $9.4946 e-03$ & $9.0274 e-03$ \\
$(0.6,0.6)$ & $2.5201 e-05$ & $7.3918 e-06$ & $8.0291 e-03$ & $1.5456 e-04$ \\
$(0.8,0.8)$ & $3.2838 e-05$ & $9.6317 e-06$ & $6.7784 e-03$ & $2.6186 e-04$ \\
\hline
\end{tabular}

correspond to $m>n$ and $m=n$. The approximate solution becomes more accurate as $m$, nincrease from the tables and figure.

Example 2. Consider the following equation:

$$
\begin{aligned}
u(x, y)= & f(x, y) \\
& +\iint_{0}^{1} \frac{x}{(8+y)(1+s+t)} u(s, t) d s d t,
\end{aligned}
$$

where $f(x, y)=1 /(1+x+y)^{2}-x / 6(1+y)$ and $u(x, y)=$ $1 /(1+x+y)^{2},(x, y) \in([0,1) \times[0,1))$.
In the first three columns of Table 3 list the absolute error for $m=n=4,8$, and 16 , respectively, and the approximate solution more accurate than the last columns of midpoint rule. The numerical results of current method compare with 2D-TFs method [10] in Table 4. We can see that the current method solutions compare quite well with 2D-TFs [10] results at different points of the domain. And Figure 2 shows the surfaces of the absolute error in a square on three different situations. When $m<n$ with $\theta_{p}=p / 10(p=$ $0,1, \ldots, 10)$, the absolute error surfaces have been obtained for $u_{m n}$, as depicted in Figure 2(a). Similarly Figures 2(b) and 2(c) correspond to $m>n$ and $m=n$. It can be observed 


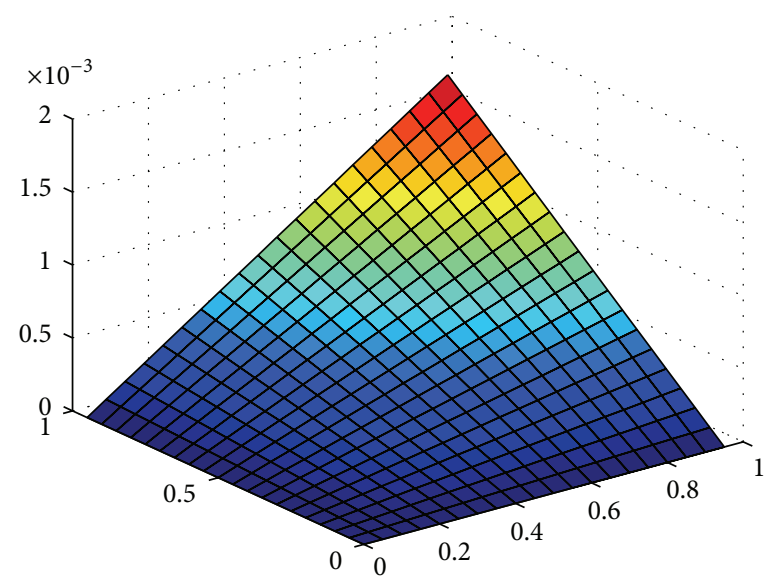

(a) $m=8, n=12$, and $q=10$

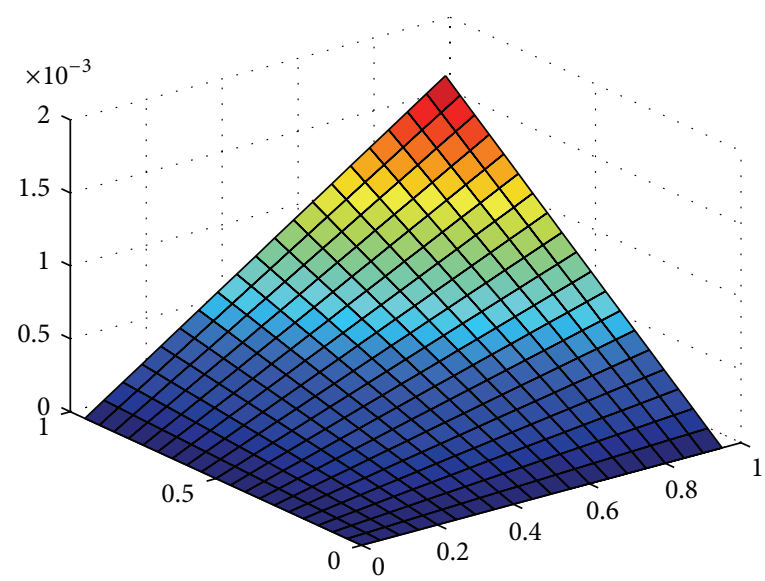

(b) $m=12, n=8$, and $q=10$

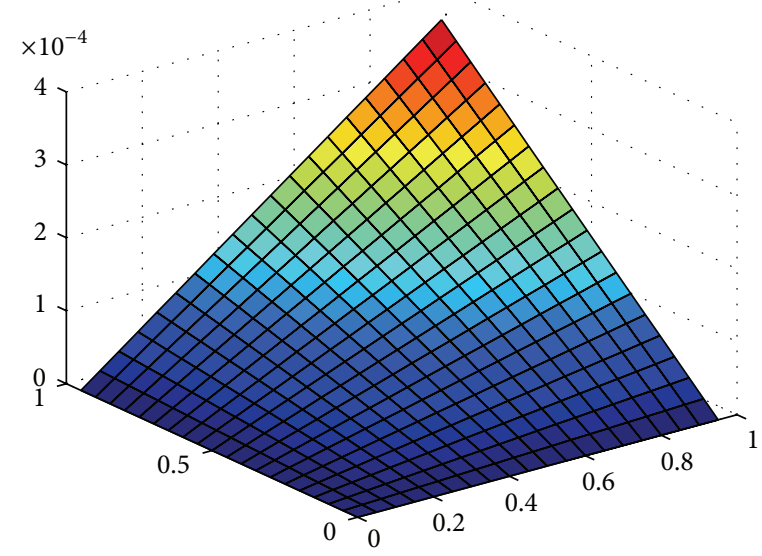

(c) $m=20, n=20$, and $q=10$

FIgURE 1: The absolute error surfaces of Example 1.

that the approximate solution becomes more accurate with respect to increasing $m, n$.

Example 3. We consider exterior boundary value problem with a potential $u(x)$, which satisfies the Laplace equation as follows:

$$
\begin{aligned}
\Delta u(x) & =0, \quad x \in D^{e}, \\
\frac{\partial u(x)}{\partial n} & =f(x), \quad x \in \partial D^{e} \\
u(x) & =O\left(|x|_{2}^{-1}\right), \\
\frac{\partial u(x)}{\partial r} & =O\left(|x|_{2}^{-2}\right),
\end{aligned}
$$

$$
\text { as } r=|x|_{2} \longrightarrow \infty \text {, }
$$

where $D^{e}=\left\{x_{1}^{2}+x_{2}^{2} \geq 1\right\}$ is region outside of the unit circle and $\partial D^{e}=\left(x_{1}, x_{2}\right)=(\cos \varphi, \sin \varphi), \varphi \in[0,2 \pi]$. The boundary condition on $\partial D^{e}$ is given: $f=\left(\left(x_{1}^{2}-x_{2}^{2}\right) /\left(x_{1}^{2}+x_{2}^{2}\right)\right) \cos \varphi+$
TABLE 5: Absolute errors of Example 3, $q=10$.

\begin{tabular}{lccc}
\hline$\left(x_{1}, x_{2}\right)=\left(2^{l}, 2^{l}\right)$ & $n=4$ & $n=8$ & $n=16$ \\
\hline$l=1$ & $3.1469 e-03$ & $4.9950 e-05$ & $1.2192 e-08$ \\
$l=2$ & $3.6585 e-04$ & $3.5763 e-07$ & $3.4102 e-013$ \\
$l=3$ & $4.4733 e-05$ & $2.7305 e-09$ & 0 \\
$l=4$ & $5.5595 e-06$ & $2.1208 e-011$ & 0 \\
$l=5$ & $6.9392 e-07$ & $1.6544 e-013$ & 0 \\
$l=6$ & $8.6708 e-08$ & $1.2906 e-015$ & 0 \\
\hline
\end{tabular}

$\left(2 x_{1} x_{2} /\left(x_{1}^{2}+x_{2}^{2}\right)\right) \sin \varphi$ and true solution $u\left(x_{1}, x_{2}\right)=x_{1} /\left(x_{1}^{2}+\right.$ $\left.x_{2}^{2}\right)$.

We obtain numerical results of the field potential by converting exterior potential problem to boundary integral equation of the second kind. In Table 5, we report the absolute error of field potential at selected points for various choices according to the present method. And Figure 3 shows the surface of the absolute error with $n=10, \theta_{p}=p / 10$ 


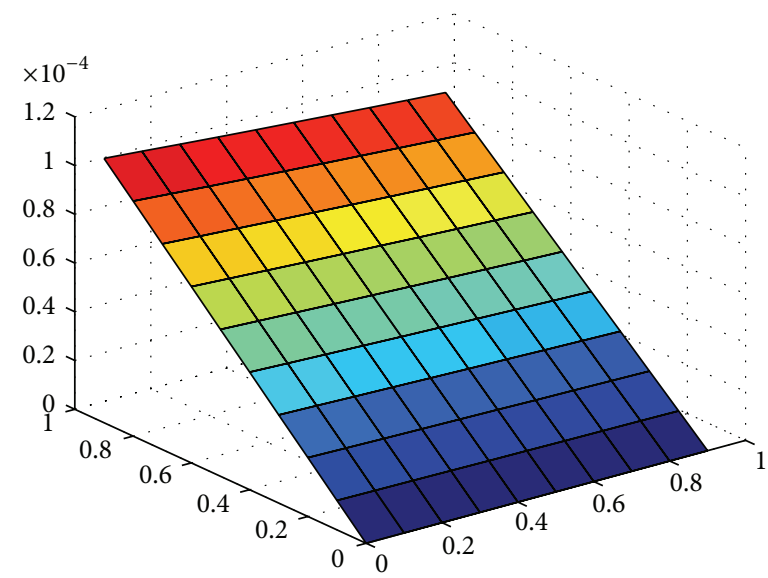

(a) $m=8, n=12$, and $q=10$

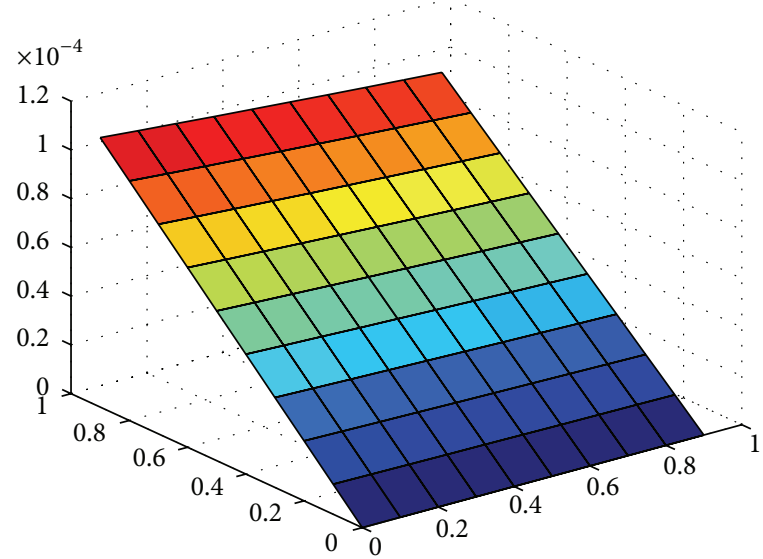

(b) $m=12, n=8$, and $q=10$

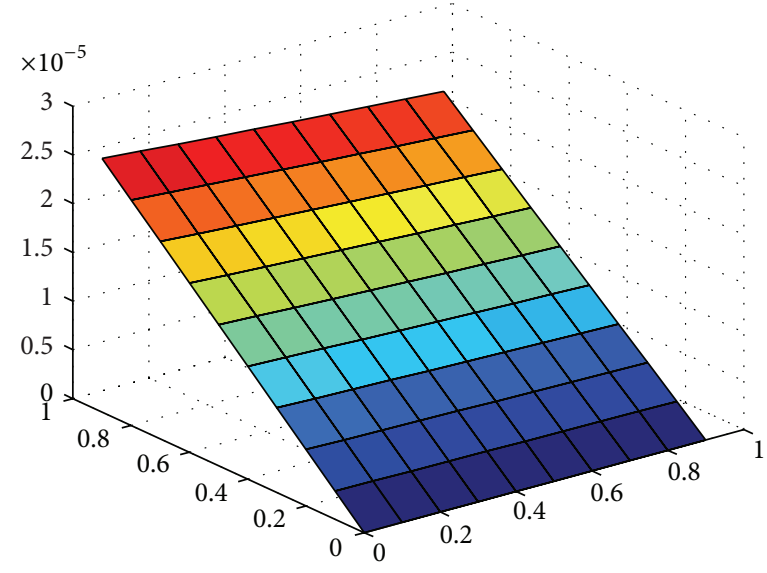

(c) $m=20, n=20$, and $q=10$

Figure 2: The absolute error surfaces of Example 2.

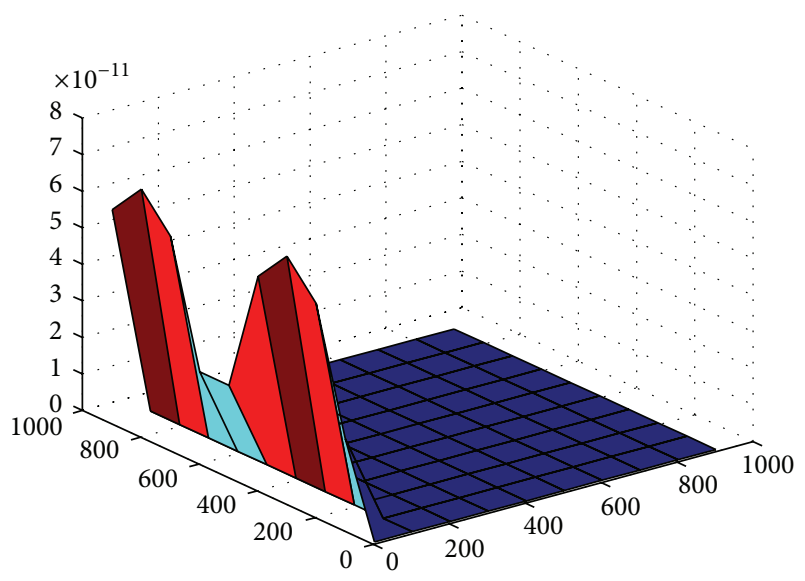

FIgURE 3: The absolute error surface of Example 3 with $n=10, q=$ 10.

$(p=0,1, \ldots, 10)$ in infinite plane. This algorithm has a high rate of convergence from Table 5 and Figure 3.

\section{Conclusions}

In this work, we state an efficient numerical solution method of the linear two-dimensional FIE of the second kind. Integral mean value theorem is utilized to reduce the computation of this problem to algebraic equation. This method is very simple and involves lower computation. In fact, this method is similar to the Nyström method. But Nyström method obtains approximate solution which is based on interpolation formula. Moreover, we can extend this approach to the multidimensional FIE and nonlinear two-dimensional FIE.

\section{Conflict of Interests}

The authors declare that there is no conflict of interests regarding the publication of this paper.

\section{Acknowledgments}

The authors are very grateful to the referees for their detailed comments and valuable suggestions, which greatly improved the paper. This work was partially supported by the financial 
support from National Natural Science Foundation of China (Grant no. 11371079).

\section{References}

[1] R. Farengo, Y. C. Lee, and P. N. Guzdar, "An electromagnetic integral equation: application to microtearing modes," Physics of Fluids, vol. 26, no. 12, pp. 3515-3523, 1983.

[2] S. Hatamzadeh-Varmazyar and Z. Masouri, "Numerical method for analysis of one- and two-dimensional electromagnetic scattering based on using linear Fredholm integral equation models," Mathematical and Computer Modelling, vol. 54, no. 910, pp. 2199-2210, 2011.

[3] K. Atkinson, D. D. Chien, and J. Seol, "Numerical analysis of the radiosity equation using the collocation method," Electronic Transactions on Numerical Analysis, vol. 11, pp. 94-120, 2000.

[4] K. E. Atkinson, The Numerical Solution of Integral Equations of the Second Kind, vol. 4, Cambridge University Press, Cambridge, UK, 1997.

[5] K. E. Atkinson, "The numerical solution of Laplace's equation in three dimensions," SIAM Journal on Numerical Analysis, vol. 19, no. 2, pp. 263-274, 1982.

[6] S. Effati and R. Buzhabadi, "A neural network approach for solving Fredholm integral equations of the second kind," Neural Computing and Applications, vol. 21, no. 5, pp. 843-852, 2012.

[7] Z. Avazzadeh, M. Heydari, and G. B. Loghmani, "Numerical solution of Fredholm integral equations of the second kind by using integral mean value theorem," Applied Mathematical Modelling, vol. 35, no. 5, pp. 2374-2383, 2011.

[8] X.-C. Zhong, "A new Nyström-type method for Fredholm integral equations of the second kind," Applied Mathematics and Computation, vol. 219, no. 17, pp. 8842-8847, 2013.

[9] Z. Avazzadeh and M. Heydari, "Chebyshev polynomials for solving two dimensional linear and nonlinear integral equations of the second kind," Computational \& Applied Mathematics, vol. 31, no. 1, pp. 127-142, 2012.

[10] F. Mirzaee and S. Piroozfar, "Numerical solution of the linear two-dimensional Fredholm integral equations of the second kind via two-dimensional triangular orthogonal functions," Journal of King Saud University, vol. 22, no. 4, pp. 185-193, 2010.

[11] A. Alipanah and S. Esmaeili, "Numerical solution of the twodimensional Fredholm integral equations using Gaussian radial basis function," Journal of Computational and Applied Mathematics, vol. 235, no. 18, pp. 5342-5347, 2011.

[12] G. Gnecco, V. Kůrková, and M. Sanguineti, "Accuracy of approximations of solutions to Fredholm equations by kernel methods," Applied Mathematics and Computation, vol. 218, no. 14, pp. 7481-7497, 2012.

[13] A. Golbabai and S. Seifollahi, "Numerical solution of the second kind integral equations using radial basis function networks," Applied Mathematics and Computation, vol. 174, no. 2, pp. 877883, 2006.

[14] Z. Avazzadeh, M. Heydari, and G. B. Loghmani, "A comparison between solving two dimensional integral equations by the traditional collocation method and radial basis functions," Applied Mathematical Sciences, vol. 5, no. 21-24, pp. 1145-1152, 2011.

[15] F. Mirzaee and E. Hadadiyan, "Numerical solution of linear Fredholm integral equations via two-dimensional modification of hat functions," Applied Mathematics and Computation, vol. 250, pp. 805-816, 2015.
[16] A. Tari and S. Shahmorad, "A computational method for solving two-dimensional linear Fredholm integral equations of the second kind," The ANZIAM Journal, vol. 49, no. 4, pp. 543-549, 2008.

[17] H.-A. Derili, S. Sohrabi, and A. Arzhang, "Two-dimensional wavelets for numerical solution of integral equations," Mathematical Sciences, vol. 6, article 5, 2012.

[18] M. Heydari, Z. Avazzadeh, H. Navabpour, and G. B. Loghmani, "Numerical solution of Fredholm integral equations of the second kind by using integral mean value theorem II. High dimensional problems," Applied Mathematical Modelling, vol. 37, no. 1-2, pp. 432-442, 2013.

[19] G. Han and R. Wang, "Richardson extrapolation of iterated discrete Galerkin solution for two-dimensional Fredholm integral equations," Journal of Computational and Applied Mathematics, vol. 139, no. 1, pp. 49-63, 2002.

[20] Y. Wang and Y. Xu, "A fast wavelet collocation method for integral equations on polygons," Journal of Integral Equations and Applications, vol. 17, no. 3, pp. 277-330, 2005.

[21] D. Occorsio and M. G. Russo, "Numerical methods for Fredholm integral equations on the square," Applied Mathematics and Computation, vol. 218, no. 5, pp. 2318-2333, 2011.

[22] G. Mastroianni, G. V. Milovanović, and D. Occorsio, "Nyström method for Fredholm integral equations of the second kind in two variables on a triangle," Applied Mathematics and Computation, vol. 219, no. 14, pp. 7653-7662, 2013.

[23] P. M. Anselone, Collectively Compact Operator Approximation Theory and Applications to Integral Equations, Prentice-Hall, Englewood Cliffs, NJ, USA, 1971. 


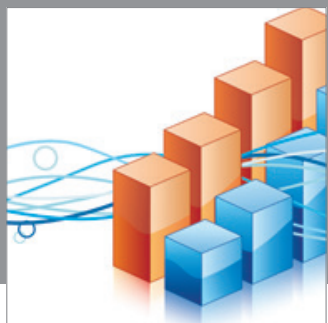

Advances in

Operations Research

mansans

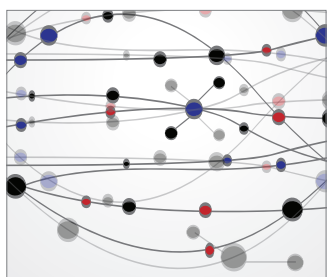

The Scientific World Journal
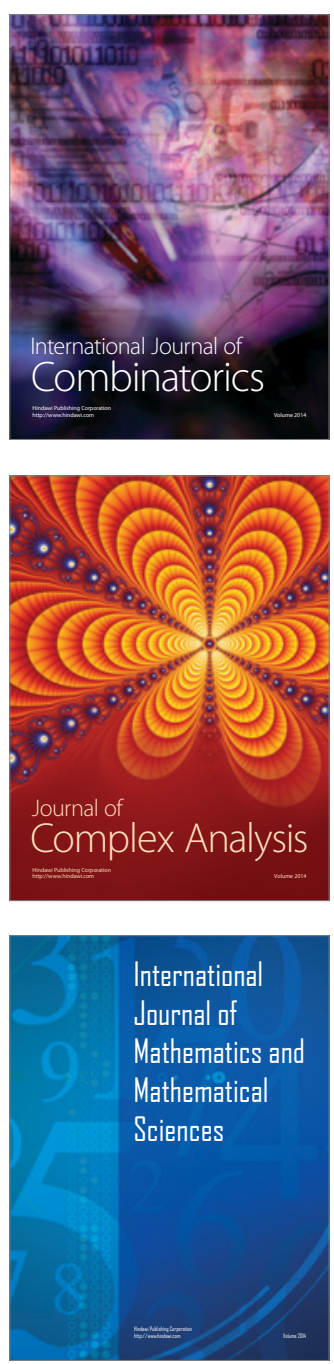
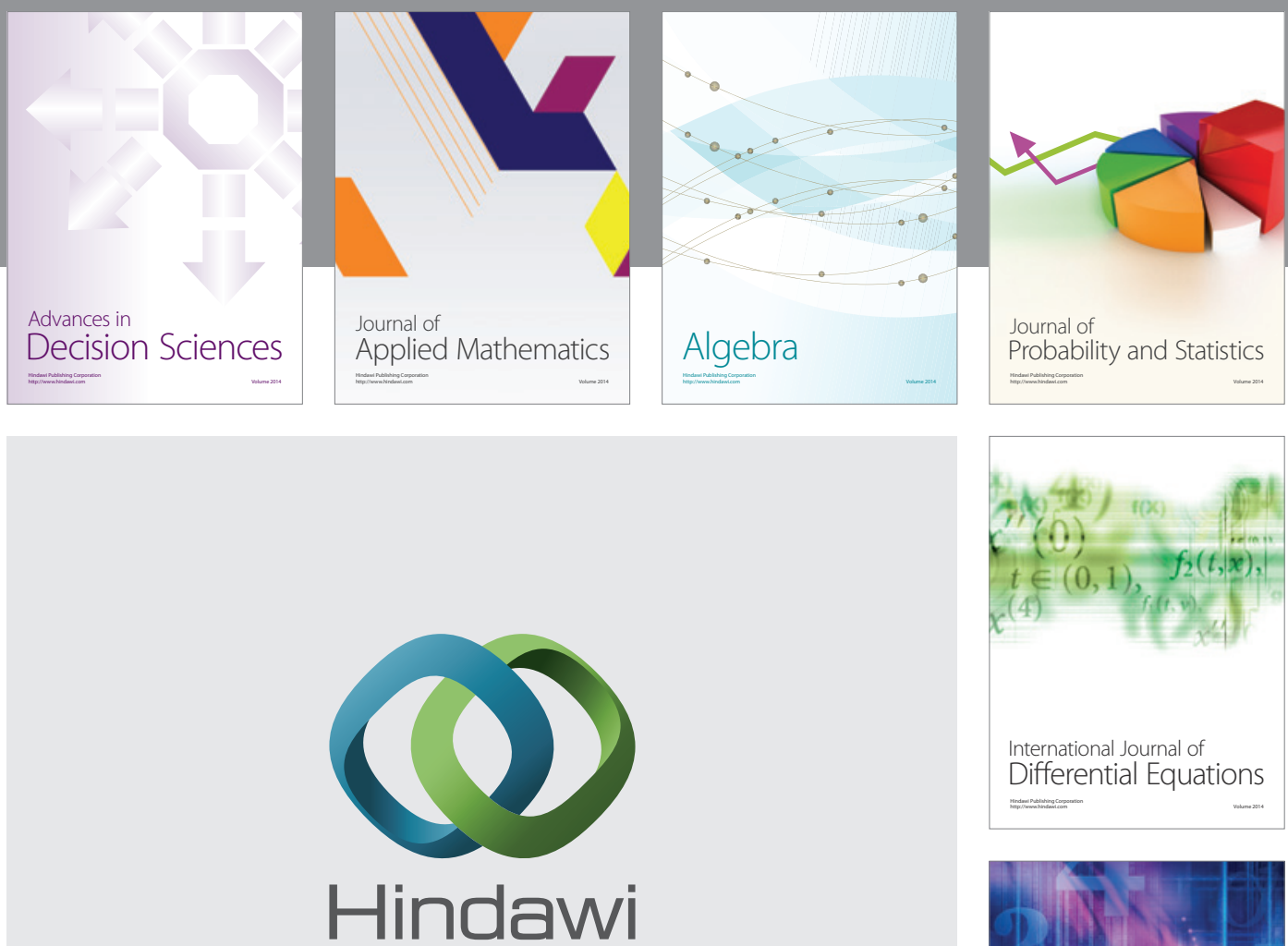

Submit your manuscripts at http://www.hindawi.com
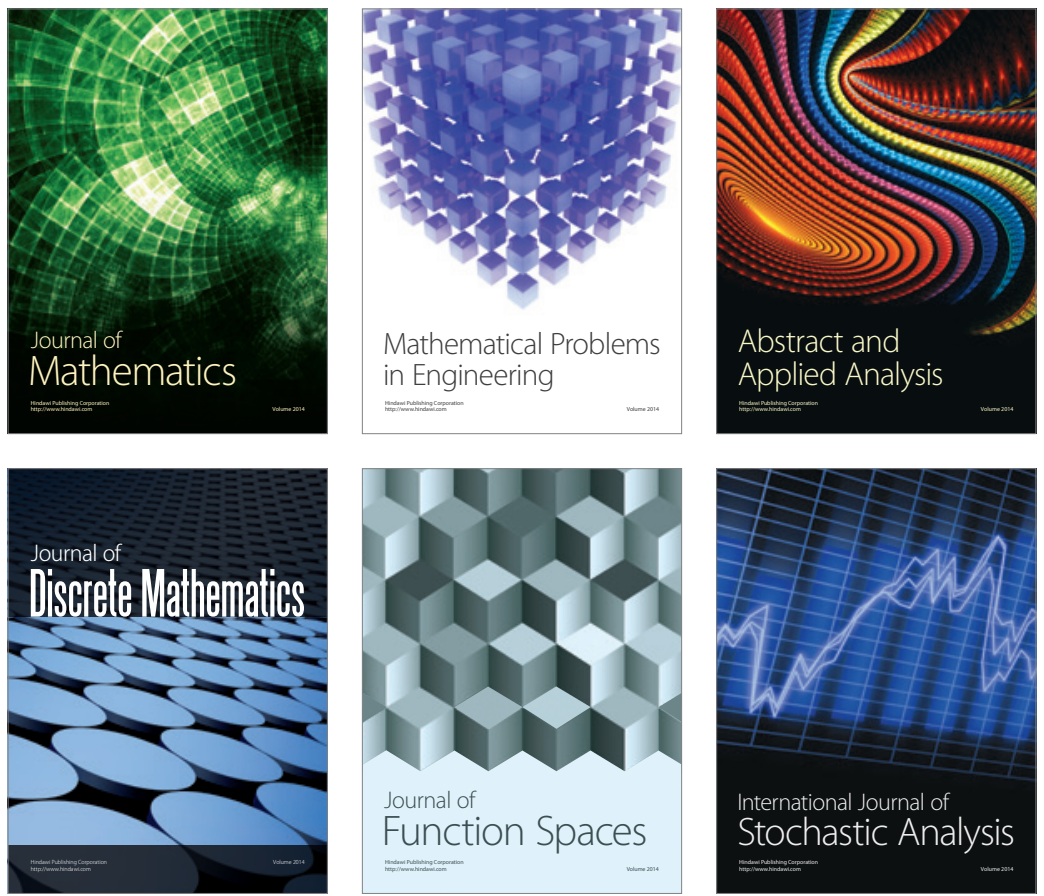

Journal of

Function Spaces

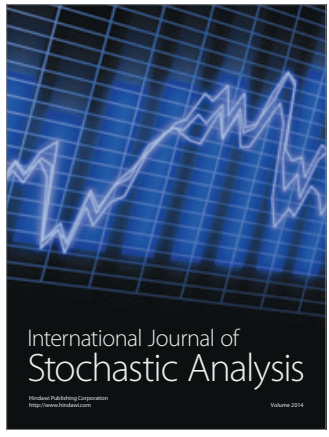

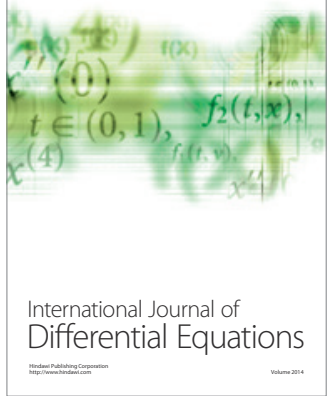
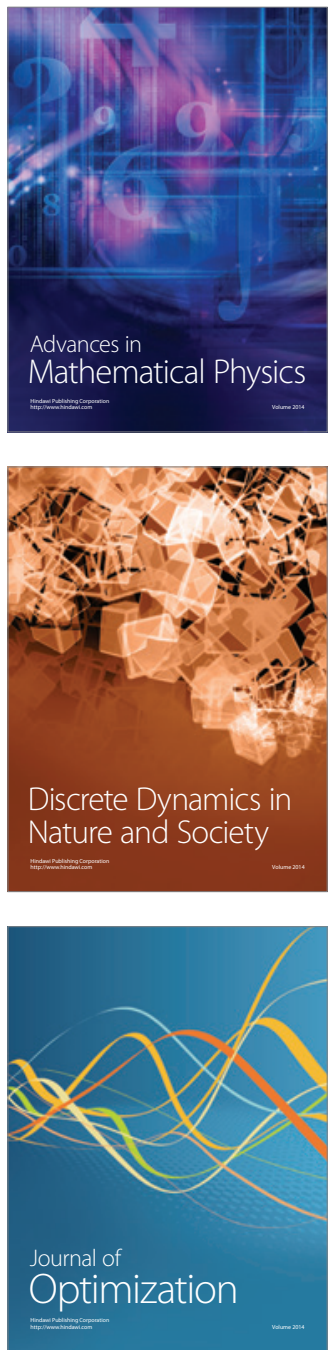